\title{
MEMOIRE ET HISTOIRE: la monumentalisation du Ghetto de Varsovie ${ }^{1}$
}

\section{Sylvia Ostrowetsky*}

L'objet de ce travail concerne les rapports que la mémoire entretient avec l'espace mais le choix du "territoire" particulier du ghetto de Varsovie est motivé par mon histoire propre et l'assurance, contrairement au discours obligé en sciences sociales sur la nécessaire distance au terrain, que cet engagement particulier me permet, sous certaines conditions, de cerner avec plus de justesse, les implications qui sous-tendent le rapport des lieux à la mémoire collective et individuelle.

Le fil rouge qui explique mon attention au ghetto de Varsovie tient donc au fait que mon métier de sociologue, teinté d'anthropologie et de sémiotique, permet d'inscrire le monument et plus spécifiquement le mémorial, sur un terrain préoccupé par l'objet artistique dans ce qu'il exprime, dénie ou manifeste de la collectivité, d'une part, au fait que les restes de ma famille forment une partie du terreau des parterres de fleurs et d'arbres qui le décorent désormais, d'autre part.

Plus largement, l'étude sur la monumentalisation du ghetto de Varsovie que je présente ici de façon partielle et forcément succincte, est, pour l'essentiel, l'occasion d'une réflexion sur le rôle social et culturel de la mémorialisation.

Fidèle à une théorie du constat, contrairement à ce que pourrait laisser croire mon engagement subjectif, il ne s'agit pas tant de prendre parti pour ou contre la monumentalisation que d'approcher son évolution au fur et à mesure des constructions destinées aux inaugurations successives dont elle fut l'objet. A ce titre, je suis persuadée que le conflit entre mémoire et histoire tel qu'il se manifeste de nos jours dans le champ du savoir, doit être largement relativisé.

* Professeur émérite, EDRESS, Université de Picardie.

Artigo recebido em 17 nov. 2004; aprovado em 15 dez. 2004. 
A propos des détournements de la mémoire, les historiens sont obligés de mettre en garde contre des opérations qui relèvent plus de l'épopée individuelle ou collective que de la vérité. Mais, à l'inverse, leurs positions critiques ont une influence, sans doute plus souterraine, sur le désir grandissant avec le temps, de respecter, au mieux, une certaine objectivité et d'échapper ainsi à une mythologie peu productive d'enseignements.

Au lieu de glorifier a priori ce qui fut en réalité, pour l'essentiel, un martyrologe rehaussé par l'acte héroïque d'une poignée de militants lors de l'insurrection de mars 1943 qui a marqué grandement les esprits et que traduisent deux des monuments les plus célèbres du ghetto, les mémoriaux - monuments ou documents - que nous avons choisis ensuite, abandonnent le schéma classique de la guerre et ses formes obligées de monumentalisation pour une description plus fidèle et élargie de ce qui a spécifié la mise à mort massive engagée par l'appareil nazi.

A ce propos, on sait quel terrible reproche certains - en Israël notamment - se sont cru autorisés de faire à ces êtres pris dans l'entreprise de dénaturation dont ils étaient l'objet privilégié en les accusant de passivité. Dans Génocide pour mémoire, Georges Bensoussan (1989, p. 140) tente d'expliquer "cette passivité des masses juives". Ce n'est certes pas le lieu ici de reprendre longuement une question qui mériterait une réflexion approfondie à elle seule, mais, à moins qu'il ne s'agisse, comme à Sobibor, de déportés exercés - soldats de l'Armée Rouge notamment, il me semble que le concept de "série", proposé en 1960 par Jean Paul Sartre dans Critique de la raison dialectique, montre, dès lors qu' on fait tout pour empêcher la moindre parole, qu'un groupe ne peut se défendre, même devant un poignée d'Allemands et d'Ukrainiens. Ainsi, la façon dont on forçait les déportés à courir et passer en file indienne, comme à Auschwitz, par ce qui était nommé "le boyau". Sans compter le mensonge pur et simple, comme la production et la consommation du même nom, la mort de masse commence par une première phase de séquencialisation préalable permettant ensuite l'enfermement dans la chambre à gaz. Ainsi que le décrit si précisément Hilberg, dans le 
film Shoah de Claude Lanzmann, cette division rationnelle mais fondée sur une psychologie rudimentaire de l'isolement en une "foule solitaire" a permis, sans aucun doute, autant qu'une disposition à la passivité attribuée couramment à la culpabilité religieuse, ce qui se passe toujours dans ces cas là (cf. Le génocide des Tutsis - voir plus bas).

Restituer à ces êtres réduits systématiquement à l'anonymat, ne fut-ce qu'un prénom, retrouver sous les décombres un objet cultuel ayant appartenu à une famille particulière, imaginer une vie intense à jamais disparue ou encore valoriser la mort volontaire d'un personnage connu que l'on a refusé d'entendre, consiste à œuvrer avec justesse à la résurgence d'une identité vouée à la disparition.

C'est pourquoi, ainsi que le font certains conservateurs des monuments historiques ou des historiens, il me parait un peu simpliste de s'opposer à ce qui constitue une des bases anthropologiques du culte des morts à laquelle aucune population n'échappe sans le payer, inconsciemment, très cher. Car, en réalité, à sa manière, la recherche de vérité est aussi une quête du passé mais fondée cette fois, sur l'indéniable de la preuve. Tout en respectant la nécessité vitale, pour nos mémoires branlantes ou figées, de la commémoration, notre monde est peut-être en train de trouver une nouvelle forme, sans doute plus respectueuse des hommes dans leur ambivalence et plus véridique dans son historicité, qui synthétise mémoire et histoire au lieu de les opposer.

\section{"Arguments ethnométhodologiques"}

Selon mon parti pris méthodologique de départ, je rappelle, ici, succinctement, que pour Harold Garfinkel, auteur des Studies in ethnomethology (1967), toute entreprise sociologique doit d'abord reconnaître qu'une enquête ne consiste pas dans la recherche d'une vérité que l'objet enquêté serait à même de fournir mais qu'elle constitue, en elle-même, une situation sociale. Ceci signifie qu'il n'y a pas moyen d'ignorer "le contexte" dans lequel elle vient s'insérer, 
d'abord, que les réponses qui seront fournies sont déjà théorisées, "réfléchies", même de manière approximative, par les locuteurs ou, comme ici, par l'enquêteur lui-même, ensuite. En conséquence, il ne sera pas question d'approcher le monde social, fut-il son produit sculptural ou architectural, comme s'il s'agissait de choses, à la manière de Durkheim. C'est pourquoi d'emblée, je déclinerai, succinctement, le contexte biographique de ma propre parole et, d'une manière plus générale, je soulignerai l'influence indirecte des réflexions que je mène depuis des années sur l'architecture, l'urbanisme et les processus identitaires.

Ainsi que mon nom, même mal orthographié - la véritable orthographe étant Ostrowiecki -, l'indique je suis d'origine polonaise: mes parents sont nés en Pologne, dans un "shetl" entre Lodz et Varsovie. Ils ont émigré en France en 1923. Comme je l'ai raconté dans un ouvrage qui leur est consacré (Ostrowetsky, 1995), ils se sont d'abord installés rue François Miron en plein Marais parisien derrière l'Hôtel de Ville, puis à Gentilly, commune rouge du sud de la banlieue parisienne.

Mon premier contact avec la Pologne, remonte à 1936. Au cours d'un voyage à Mogelnicka avec ma mère, je rencontrai l'essentiel de ma famille restée en Pologne, ma grand-mère et mes oncles notamment, dont je garde - je n'avais pas trois ans - , ainsi que du village et de la maison familiale avec son poêle et ses lourdes tentures, un souvenir très précis.

Second contact: une carte postale de la Kommandantur datée de 1942, avec un tampon de la Croix Rouge, annonçant que ma grandmère, ma tante et ses deux enfants étaient morts de faim (sic!) dans le ghetto de Varsovie. En réalité, à part un oncle engagé dans l'Armée Rouge, toute ma famille restée en Pologne a disparu dans le ghetto ou à Treblinka.

Le troisième souvenir est constitué par une photo de mon père datant des années 50. Président de "La Renaissance Juive" qui 
rassemblait les anciens de Mogelnicka et d'un village voisin, tenant de la main droite le drapeau de la Société de Secours Mutuel, on le voit debout, figé par l'émotion, devant la sculpture de Natan Rapaport, monument le plus important du ghetto.

Le quatrième souvenir date de 1969: envoyée par le District de la Région de Paris où je travaillais à la mise en place des Villes Nouvelles, on m'amena voir ce même monument que je trouvais très “stalinien”. Universaliste, je voulais ignorer, comme beaucoup d' autres à cette époque, la spécificité de la mort juive.

Le dernier contact remonte à juin 2000 lorsque je fus invitée en tant que Professeur des Universités par l'Académie des Sciences de Varsovie à faire deux conférences dont l'une portait sur l'identité culturelle, nationale et sociale.

Lors de mon séjour en 1969, la jeune interprète m'avait demandé pourquoi je ne parlais pas polonais avec un nom pareil... Je lui avais répondu que mes parents l'utilisaient entre eux quand ils ne voulaient pas que les enfants comprennent et sinon qu'ils parlaient quotidiennement le yiddish. Elle avait alors passé son doigt sur l'arête de mon nez en disant: "pourtant cela ne se voit pas"...

Mais cette dernière fois, quel accueil! Un jour, connaissant ma formation d'historienne et mon goût pour l' architecture, une collègue, d'origine chrétienne, me fit visiter les églises baroques de la vieille ville presque toutes reconstruites à l'identique à partir d'estampes comme chacun sait. Le lendemain, Andrzej Leder, dont la mère, émigrée en France, était retournée en Pologne par conviction politique, me fit visiter le ghetto. Fleuri, ensoleillé, qui peut imaginer en parcourant ce lieu, l'enfer que ses habitants ont vécu ici?

Remarque qui corrige peut-être le relativisme exacerbé des ethnométhodologues: mon propos concerne, je le rappelle, non pas une enquête auprès des hommes - créateurs, acteurs sociaux divers ou usagers - mais les monuments eux-mêmes. Ce parti-pris explique la raison pour laquelle je me suis inspirée d'une méthode descriptive qui n'échappe pas, elle non plus, à l'interprétation mais qui permet 
d'articuler ce que l'on nomme en général forme et signification ou, pour utiliser le vocabulaire du sémioticien Louis Hjelmslev dont je me sers ici, "expression" et "contenu", de façon systématique.

\section{Descriptif des monuments et documents choisis ${ }^{2}$}

J'ai privilégié non seulement les quatre mémoriaux les plus importants, c'est-à-dire les plus fréquemment photographiés et cités mais aussi deux objets qui me semblent hautement significatifs d'une attitude esthétique et mémorielle plus contemporaine.

1 - Le monument dont je viens de parler du sculpteur Natan Rapoport et de l'architecte Léon Marek Suzin dédié au soulèvement du ghetto de 1943. Inauguré en 1948, il renvoie, sans conteste, à l'Holocauste décrit dans l'Ancien Testament renouvelant le sacrifice de Massada devant l'envahisseur romain et participe ainsi d' un mythe ancestral. $^{3}$

Ce monument de onze mètres de haut, dont les sculptures sont en bronze, a été construit, à sa base, avec des matériaux qui devaient servir à l'érection d'un monument consacré à Hitler! Il se présente sous la forme d'une pyramide tronquée ornée, sur la face avant, d'un haut-relief représentant l'insurrection avec au centre un jeune homme identifié à Mordechaï Anielewicz, commandant de la révolte alors âgé de vingt-trois ans, et, sur la face arrière, d'un bas-relief en granit, où le peuple en exode est figuré par douze personnages harassés représentant les douze tribus d'Israël. Le monument est situé sur une vaste esplanade au centre de l'ancien quartier juif bordé, depuis la fin de la guerre, par des immeubles récents.

Dans la revue Pardès, James E. Young (1991) décrit l'histoire de la construction du monument et rapporte son entretien avec le sculpteur lui-même et un propos qui oscillait entre "la mythologie prolétarienne de l'époque stalinienne" et "les images typique des juifs en exil".

Au moment de passer de la maquette de plâtre au bronze, dépourvu de tout moyen matériel en Pologne, Rapoport se rend à 
Paris. Il prend alors conscience que sa place dans l'art contemporain est marginale. Mais, persuadé de sa mission de témoin, il tient à "montrer au peuple polonais qui nous étions vraiment" (Young, 1991, p. 68) et conserve le parti-pris d'une forme structurée et figurative.

Un mois avant l'inauguration officielle à Varsovie, quand le groupe sculptural est présenté à Paris, le doyen des critiques d'art parisiens proclame: “C'est une œuvre superbe (...) d'un intense pathos, vivante, puissante et d'un équilibre magistral" qui renvoie à Rodin, surtout à Bourdelle sans pour autant être archaïque...

2 - "La route du souvenir", constituée de dix neufs blocs de syénite noire, est disposée selon un parcours qui part du monument de Rapoport et aboutit à la place de transbordement l'Umschlagplatz - pour le camp d'extermination de Treblinka. Construite en 1988 par Zbigniew Gasior, Stanislaw Jankowski et Marek Moderau pour le quarante-cinquième anniversaire du soulèvement du ghetto, ${ }^{4}$ cette nouvelle proposition relève d'une conception différente du mémorial.

Chaque bloc de pierre est singulièrement consacré à l'un des héros du ghetto dont on veut honorer la magnanimité. Nom et dates gravés sur le côté et description succincte de leur rôle ou de leur responsabilité militante sur la surface plane et lisse à l'horizontale, chacun d'eux est valorisé expressément. ${ }^{5}$ Il s'agit ici de suivre un chemin où la gloire a certes perdu ses visages mais gagné ses héros. ${ }^{6}$ On remarquera que ce choix de l'abstraction, observe, peut-être involontairement, l'interdit de la représentation spécifique de la religion juive (...et musulmane).

3 - Avec l'architecture de l'Umschlagplatz, il s'agit cette fois d'un mausolée consacré aux masses condamnées à l'extermination... Alors que le monument de Rapoport veut glorifier un peuple, que "le chemin du souvenir" consacre des hommes d'exception, ce dernier monument marque une évolution différente du mémorial.

Lorsque les nazis décidèrent la liquidation du ghetto, ceux parmi les juifs qui affluèrent à l'Umschlagplatz pour une miche de pain et 
un pot de marmelade, faisaient un choix innommable entre mourir de faim dans le ghetto ou manger une dernière fois en allant à une mort certaine. C'est en arrivant à l'Umschlagplatz, écrit Marek Edelman, qu'ils comprenaient vraiment ce qui les attendait. ${ }^{7}$

Cette architecture, ${ }^{8}$ inaugurée le 18 avril 1988, œuvre d'Hanna Szmalenberg et de Wladyslaw Klamerus, ${ }^{9}$ se présente comme un grand parallélépipède recouvert de marbre blanc ceinturé d'une ligne noire. Sur le devant, une ouverture, surmontée d'un demi-cercle faisant à la fois tympan et linteau, permet de pénétrer à l'intérieur d'un édifice sans toiture qui contient des plaques écrites en hébreu, polonais, yiddish, anglais et trois cent cinquante prénoms égrenés sur les murs. Cette première ouverture fait face à une sorte de porte cochère entrouverte dont le sommet adopte une même forme en demi-cercle mais blanche en opposition au premier tympan noir. Comme si l'ouverture principale était en réalité la translation d'une porte-glissière, on trouve, à droite, un pan de mur de même taille que l'ouverture. Ainsi, les deux fentes qui se font face, l'une large, à l'entrée, l'autre, étroite, à l'arrière, et ce pan isolé, donnent exactement l'impression de la mobilité propre aux portes des wagons à bestiaux. ${ }^{10}$ En même temps, de façon anthropomorphe, entièrement drapé dans un immense châle de prière (le talith) qu'évoque la bande noire, c'est le mémorial en son entier qui officie. ${ }^{11}$

4 - C'est dans la veine de l'indice ou de la trace, que les habitants, essentiellement chrétiens, des quartiers de Muranow et Mirow, en faisant des travaux dans leur cave ou lors de l'effondrement des murs - car dans l'immédiate après guerre, la construction s'est faite à même le terrain qui avait été arasé par les déportés amenés sur place après la révolte - découvrent des objets conservés dans les ruines. Jaciek Leociak, chercheur, y a trouvé, entre autres, une timbale gravée de lettres hébraïques. C'est peut-être ${ }^{12}$ lorsqu'ils s'engagent dans cette quête, lorsqu'ils refont, tel Jaroslaw Marek Rymkiewicz (1998), polonais chrétien, les mêmes parcours, les mêmes stations, c'est à ce moment là, peut-être, qu' ils considèrent enfin, ces importuns voisins qu'ils côtoyaient depuis des siècles, comme de vrais Polonais. Ces quelques objets meurtris, cette timbale, sont le seul témoignage de la présence du ghetto, sa seule mémoire vive. 
5 - En 1986, l'Association pour la Protection des Monuments Anciens de l'Ancienne Varsovie tente de préserver ce qui reste de la rue Prozna qui donne sur le square Grzybowski. Avec ce reste de mur fait de briques superposées, il ne s'agit plus, ni de la révolte du ghetto, ni, par un monument, d'honorer des victimes mais de conserver un témoignage de l'époque qui a précédé la "catastrophe” (Shoah).

Cette portion de rue ${ }^{13}$ constitue l'un des rares souvenirs du temps où des citadins se côtoyaient dans l'affairement plus ou moins joyeux mais affirmatif qu'Ezra Park, fondateur du premier enseignement de sociologie urbaine aux États-Unis et élève de Georg Simmel, a nommé une "société de passants". Ici, pas de gloire, pas de héros, pas de noms ni, à défaut,t de prénoms, mais l'évocation d'une ville que l'on sait dense, garnie de petites échoppes d'où émanaient les odeurs des cornichons en saumure et des harengs marinés. Cette préservation tente de sauvegarder la mémoire d'une période révolue, un temps arrêté. ${ }^{14}$

Cette attention portée à une ruine relève de ce que Aloïs Riegl (1984) nomme le "culte moderne de l'authenticité", qui sera, de façon parallèle, rejointe, dès 1914, par Duchamp. En effet, si ce dernier désacralise l'art en exposant un porte-bouteilles dans un espace muséal, c'est bien pour démontrer la volonté d'en finir avec la séparation élitiste de l'œuvre au bénéfice d'une qualification historique et anthropologique des modestes objets de la vie quotidienne. Encore que la charge affective soit sans commune mesure, en s'inspirant de Michel Melot, ${ }^{15}$ on peut dire ainsi que, tel un "ready-made", ce morceau de rue est un "objet utilitaire dont on détourne l'usage pour en faire un objet emblématique" (p.18).

Ce même raisonnement explique, sans doute, que le choix de wagons qui avaient servi au transport des déportés aient pu être transformés en mémoriaux comme à Drancy, au camp des Milles, à Milly-la-Forêt ou à Yad Vashem en Israël. Avec la rue Prozna, une partie de la ville est devenue, tout à la fois, emblème et document. ${ }^{16}$

6-Rue Zamenhofa, enfin, face à un mur vitrifié qui renvoie en miroir la fluidité de la rue, mur gravé de son dernier message, en 
yiddish et polonais, se trouve la "compression" à la mémoire de Shmuel Zygielbojm dont les auteurs sont Hanna Szmalenberg (qui a participé avec Klamerus à la création du monument de l'Umschlagplatz), et Marek Moderau, l'un des auteurs du "chemin du souvenir". Hanna Szmalenberg a expliqué personnellement à Andrzej Leder - mon informateur polonais - qu'ils avaient décidé de mémorialiser Shmuel Zygielbojm (il devait au départ, faire partie du "chemin du souvenir"), "d'une façon particulière". ${ }^{17}$

Il s'agit d'une dalle de pierre brisée par une masse, et que l'on a recomposée en laissant des rigoles entre les morceaux, tel un puzzle mal ajusté. Le mémorial est posé sur les pavés à même la rue. Ce geste constitue une allégorie de l'impuissance à "briser" le mur du silence. "Démoralisé, découragé, abattu, désespérant d'aboutir à un quelconque résultat lorsqu'il constate que la conférence angloaméricaine des Bermudes consacrée aux réfugiés (19-30 avril 1943) n'envisage pas la moindre mesure concrète (... ) S. Zygielbojm prend la décision de mettre fin à ses jours dans l'espoir que la nouvelle de son suicide permettra du moins d'électriser l'opinion mondiale..." ${ }^{18}$

Sans entrer dans le détail ici, cette compression correspond au passage de l'art moderne à l' art contemporain qui récuse l'objet d'art au profit de l'acte.

En réalité, c'est toute l'entreprise mémoriale qui, par définition, est saturée par cette volonté délibérée d'engager le visiteur dans une visite rituelle à l'instar de l'entreprise esthétique qui se veut novatrice de notre époque. ${ }^{19}$

\section{Légitimité de la monumentalisation mémorielle}

La façon d'approcher un lieu, dit Maurice Halbwachs (1997, p. 52), est différent pour l'historien, le commerçant, le peintre... Il ajoute: "Il ne suffit pas de reconstituer pièce à pièce l'image d'un événement passé pour obtenir un souvenir. Il faut que cette 
reconstitution s'opère à partir de données communes qui se trouvent dans notre esprit aussi bien que dans ceux des autres" (p. 63).

Remarque particulièrement pertinente quant à notre objet: "C'est que l'histoire, en effet, ressemble à un cimetière où l'espace est mesuré, et où il faut, à chaque instant, trouver de la place pour de nouvelles tombes" (Halbwachs, 1997, p. 100). Non que l'ancien espace du ghetto soit réductible à un simple cimetière mais il constitue désormais une sorte de Téménos; espace où viennent s'inscrire, avec le temps, des gestes de plus en plus fidèles à l'histoire, de plus en plus distancés du sentiment de culpabilité de n'avoir su se défendre, sans perdre pour autant sa valeur de sacralité engendrée, comme aurait dit Riegl, par le culte de l'objet authentique conçu comme une véritable relique du monde moderne. De toutes les façons, “ce n'est pas sur l'histoire apprise, c'est sur l'histoire vécue que s'appuie notre mémoire" (p. 105). D'où le rôle primordial du rituel, de l'art, pour sensibiliser les esprits qui ne l'ont pas vécue directement. Il s'agit de créer une sorte de milieu favorable à la constitution d'une conscience collective.

Pour celui qui l'a vécu au contraire ou auquel ses parents ont pris soin de le transmettre comme histoire familiale ou collective, le temps de la catastrophe ne passe pas. Corps et âme sont à jamais inscrits dans ce temps devenu un présent perpétué de la douleur, un temps hors du temps. Dans le nu de la vie, ouvrage auquel nous avons fait allusion plus haut, Jean Hatzfeld (2000) traduit de façon magistrale ces "récits des marais rwandais: "Ce qui s'est passé à Nyamata, dans les églises, dans les marais et les collines, ce sont des agissements surnaturels de gens bien naturels" (p. 72). Mais, comme le dit encore un proverbe rwandais: "les pleurs d'un homme coulent dans son ventre". A travers la parole, le monument et le rituel qu'il induit, il s'agit que ces larmes sortent et deviennent des pleurs communicables. Le mémorial transforme ce temps arrêté du trauma en un temps du souvenir. Un lieu consacré pour faire retour, afin que la communauté revive mais aussi remette la vie à sa place ${ }^{20}$ et permette une réconciliation de l'individu souffrant avec une société accueillante. 
La logique de l'art ainsi que celle de l'écriture, fut-elle "savante", est régie, en majeure partie, comme toute existence collective, dirait Gregory Bateson (1977), par le "double bind": dire et taire, à la fois, l'obscénité pudique du témoignage...

A propos de Drancy, transformé en camp d'internement après la Rafle du 20 août 1941, et où séjournèrent plus de 100.000 Juifs en transit pour Auschwitz, ${ }^{21}$ Françoise Choay s'interroge. Lors d'une émission à France-Culture le 0901 02,22 elle se déclare "formellement, contre le classement (aux Monuments Historiques ) de La cité de la Muette... du double point de vue de la Shoah d'une part et de l'intérêt que son architecture et son urbanisme pourraient avoir pour l'histoire, de l'autre". Cette proximité entre mémorial et Habitation à Loyer Modéré (HLM) lui semble sans doute indécente mais surtout la monumentalisation en elle-même ne peut engendrer, selon elle et bien d'autres, qu' un "tourisme de la mort et des gains financiers indignes". 23 Reprenant l'exemple de Verdun, elle considère que "les seuls monuments authentiques que notre époque ait su édifier ne disent pas leur nom et se dissimulent sous des formes minimales et non métaphoriques". ${ }^{24}$

Dans plusieurs ouvrages et surtout en tant qu'organisateur de l'exposition Les arts de la commémoration: mémoriaux de l'holocauste dans l'histoire (1994), James E. Young prétend que le temps est plus fidèle à l'esprit de la commémoration juive que les lieux qui risquent de finir en parcs à thèmes. ${ }^{25}$ D'où sa valorisation de l'utilisation d'une cassette-vidéo sur les deux minutes de silence de Yom Hashoah (journée commémorative de la Shoah) en Israël.

Il me semble que seul le parti-pris de l'art moderne qui récuse la notion d'œuvre au profit de l'éphémère peut justifier une position aussi tranchée. ${ }^{26}$ Le choix d'une dimension - le temps - plutôt qu'une autre - l'espace - ne peut éviter une opération qui tient à nos sociétés de masse et à laquelle on n'échappe que par l'action et la vigilance.

Surtout, l'attitude esthétique contemporaine, qui place le rituel au centre de ses préoccupations, invite à une nouvelle modalité du rite intégré au monument lui-même, que n'a pas perçu James E. 
Young. Non plus une œuvre figée à travers sa matérialité même, mais un engagement physique face, ou plus encore, au sein de l'objet esthétique. Emplir l'objet de sa propre présence comme si elle faisait partie de l'œuvre même. L'Umschlagplatz n'est œuvre que lorsque ses visiteurs y pénètrent, que le passant fait tomber, par inadvertance, devant la compression dédiée à Zygielbojm, le gobelet de carton qu'il tenait dans sa main, en un dernier geste d'abandon.

C'est à travers ce parcours dans l'espace de ce qui fut le ghetto que j'ai pu revivre, tel un film rejoué à l'envers, l'insondable gravité de ce que j'ai traversé, que je réalise et comprends le sens de mes propres engagements.

\section{Proposition de classement}

Pour plus de clarté théorique, nous proposons de différencier (alors qu'ils sont en fait liés dans le travail des artistes), expression sculpturale, architecturale et urbaine d'un côté et contenu de l'autre. On obtient ainsi:

\begin{tabular}{|c|c|c|c|c|}
\hline & Objet & \multirow{2}{*}{ Expression } & \multirow{2}{*}{ Usage } & \multirow{2}{*}{ Contenu } \\
\hline & Monument/document & & & \\
\hline 1 & sculpture de Natan Rapoport & Figuratif & Glorification & Collective \\
\hline 2 & "chemin du souvenir" & Abstraction & Heroïsation & Individuelle \\
\hline 3 & "Umschlagplatz" & Réplique & Anonymat/Identification & Universelle \\
\hline 4 & objet cultuel (timbale gravée) & Symbole & Culturel (relique) & Singulière \\
\hline 5 & rue Prozna & $\begin{array}{l}\text { Synecdoque et } \\
\text { Métonymie }\end{array}$ & Societal & $\begin{array}{l}\text { Continuite } \\
\text { temporelle }\end{array}$ \\
\hline 6 & Compression à S. Zygielbojm & Métaphore & Suicide altruiste & Rupture \\
\hline
\end{tabular}

Selon le schéma structural banal, au Plan de l'Expression, pour reprendre la terminologie hjelmslévienne, la figuration s'oppose à l'abstraction, la réplique du wagon s'oppose à l'authenticité de la timbale. Du côté du Plan du Contenu cette fois, la gloire d'un peuple conçu comme collectivité s'oppose à l'héroïsation individuelle tout 
comme la vie collective de l'ancien ghetto dans sa continuité temporelle s'oppose à la discontinuité suicidaire d'un individu.

Cependant, au delà de ce jeu différentiel, la volonté générale de donner au moins un nom à ceux qui ont disparu dans un total anonymat, de trouver dans une malheureuse timbale, la preuve intacte d'une existence déchue, fait de l'objet non seulement une preuve mais, à sa manière, une relique. Mémoriaux ou documents participent d'un nouveau rituel: un rituel de vérité.

La rue est une partie du tout (synecdoque) de la vie urbaine dans son ensemble telle qu'elle existait avant la destruction mais ne valorise que le contenant par rapport à un contenu (métonymie), celle des citadins disparus. La rue Prozna s'oppose, dans sa continuité urbaine à la métaphore langagière ("une vie brisée") de la rupture du suicide altruiste. ${ }^{27}$

Ce classement n'a pas la prétention de dire "la vérité" des mémoriaux mais de montrer comment, plus ou moins consciemment, chacun a exprimé une facette de cette vérité historique. De monument en monument, cette dernière s'est mise en place sans que l'on puisse dire, du point de vue de son fonctionnement collectif et en relation avec son contexte social et politique, lequel est plus faux ou plus vrai que l'autre. En sociologie, toute manifestation est d'abord à comprendre - ce qui ne signifie pas à excuser ou approuver - mais jamais à juger. Soucieux de mettre fin à la grandiloquence du réalisme socialiste voué à ce que Jean François Lyotard (1979) nomme "les grands récits", les propositions ont, progressivement, adopté une posture où l'éthique rejoint l'histoire.

Mais il y a plus; chacun rejoint dans sa manifestation esthétique les valeurs fondamentales qui structurent une culture et une société : celle du collectif et de l'individuel, de l'universel et du singulier, du temps continu et du temps fléché. Ce sont ces grands invariants qui viennent se figurer là selon les périodes où le collectif est aussi le temps de l'histoire comme celui de la révolte du ghetto, où l'acte individuel acquiert le sens d'une interrogation sur la responsabilité collective, où la négation de l'homme générique telle qu'elle se 
manifeste dans chaque génocide, signe l'universalité de la diversité humaine dans sa revendication identitaire... Ainsi, ce n'est pas par hasard si l'on ne considère au départ qu'un peuple en armes et si l'on s'interroge à la fin sur le sens d' un acte individuel face à ce qui lui est apparu comme indifférence collective... Ce qui est remarquable, c'est que les valeurs essentielles qui fondent tout société et qui en construisent les liens eidétiques viennent se matérialiser ainsi concrètement au fur et à mesure que l'histoire avance. Autant dire que la structure ne joue pas contre l'histoire mais avec elle.

Dans Ce que l'art fait à la Sociologie, Nathalie Heinich (1998) considère que les sociologues ont droit à deux options en ce qui concerne le fait artistique: "la première consiste à expliquer les actions, les représentations ou les productions des acteurs..." La seconde "consiste non plus à expliquer... mais à expliciter, à mettre en évidence la logique interne, la cohérence des systèmes de représentations".

Sans discuter ici ce terme de "représentation" qui, d'un point de vue épistémologique, pose bien des problèmes (Ostrowetsky, 1984), on ne situera notre entreprise, ni d'un côté, ni de l'autre de ces pôles mais dans leurs liens consubstantiels. Comme nous l'avons affirmé d'emblée, il ne s'agit, ici, ni d'une sociologie des acteurs, ni - coupure bien idéaliste - de leurs images mentales plus ou moins élaborées, mais de leurs productions matérielles, esthé(s)tiques et ce qui s'y inscrit de particularité culturelle, de différenciation sociale et d'orientation du sens.

La performativité de l'esthétique contemporaine est à la fois un appel à un mode de connaissance spécifique du sensible, ainsi que le dit Hans Georg Gadamer (1996), ${ }^{28}$ mais aussi à la constitution du Sujet collectif et individuel en tant qu'acteur de sa propre vie, futelle tragique. C'est pourquoi les mémoriaux sont si importants à nos yeux. Ils sont l'inverse même de la jouissance marchande, de son caractère foncier d'amnésie, d'obsolescence et désidentification constamment déniée. Dans le meilleur des cas, il en sont leur transgression: une mémoire vivante. 
L'art mémorial pose au sociologue une question essentielle concernant à la fois le sens de l'art et du plaisir - euphorique ou disphorique - auquel il appelle lorsqu'il s'agit, comme c'était le cas dans la tradition des pleureuses, de toucher les âmes et les corps. Il pose également la question du consensus à un moment historique, le nôtre, où la diversité des positions culturelles et idéologiques est la norme. Il pose enfin, comme le souligne également Nathalie Heinich, la question qui ne cesse de traverser le monde de l'art entre la création singulière et son destinataire collectif. De ce point de vue, certes, la sociologie a à apprendre de l'art, mais l'art, contemporain en particulier, a, aussi, et peut-être surtout, à apprendre de la sociologie. Que l'on se souvienne de cette manifestation des sans-papiers de 1995 où des acteurs de rue au regard hagard, juchés sur des échasses, agitant un immense drapeau noir, blanc, gris, une valise de carton à la main, ont surplombé la foule durant tout le temps la manifestation, place de la République et dans la rue, donnant à voir et à "vivre" à chacun, cet effarement de l'homme dépourvu d'identité. ${ }^{29}$

$\mathrm{Au}$ chapitre V de La mémoire collective, Maurice Halbwachs (1997), se référant à Auguste Comte, insiste sur le rôle de la fixité des lieux dans la constitution de la mémoire. Dans ce sens, ce n'est pas pour rien que les Allemands ont diminué progressivement l'espace $\mathrm{du}$ ghetto pour finir par le raser. On supprime aussi un peuple en détruisant ses cadres inscrits dans l'impassibilité des pierres ( $\mathrm{p}$. 191). A une personne qui me demandait comment je pouvais parler du ghetto alors qu'il n'en reste pratiquement rien, j'ai répondu, notamment grâce à Halbwachs et Hans Georg Gadamer, que c'est au ghetto tel qu'il n'est plus mais tel qu'il insiste, que les monuments rendent hommage.

La mémorialisation nous informe, comme l'on l'a vu, à sa manière, sur un mode de connaissance que la sociologie a trop ignoré sans doute, mais, à son tour, l'art s'imprègne de l'enseignement du sociologue ou de l'anthropologue qui sait bien que l'on ne fait pas un "travail" de mémoire avec les seules vitrines des musées. Ce que l'art moderne a compris c'est la nécessité structurelle du rite qui ne récuse en aucune manière la permanence des lieux et les architectures consacrées. 
Comment mesurer l'acte de langage, véritable "performatif" visuel et sensoriel que le monument ou toute œuvre implique? Dans certains de nos travaux, en suivant nos enquêtés à la trace et selon une stratégie méthodologique de participation, nous avons pu repérer ces moments privilégiés où la forme d'une architecture, le dispositif général d'une rue, une fenêtre derrière laquelle l'on a vu se dérouler un événement dramatique, font "acte". ${ }^{30}$

Les mémoriaux sont une tentative pour soutenir (la métaphore architecturale n'est ici pas de trop) l'insoutenable. A l'échelle d'une collectivité de trois cent mille personnes, le ghetto fut le règne d'un "régime qui s'accomplit" dans le "dépérissement". ${ }^{31}$ Soutenir l'insoutenable signifie insister, persister, tenter d'atteindre le point extrême qui mine le vivant. Il ne s'agit, encore une fois, nullement d'une simulation mais d'une adresse des disparus aux survivants.

Les mémoriaux tentent une aporie douloureuse: témoigner de l'intémoignable. Pour réaliser cela, ce n'est pas seulement l'archive, la preuve qui compte, mais ce que seuls peut-être les artistes peuvent faire : donner un visage à qui n'en avait plus, une volonté de fer à ces jeunes gens faméliques non identifiables, la beauté de l'éclat à celui qui s'est offert à la mort dans un dernier cri.

Malgré sa nécessaire insuffisance, le mémorial s'élève pour que jamais aucun S.S. ne puisse dire: "Et même s'il devait subsister quelques preuves, et si quelques uns d'entre vous devaient survivre, les gens diront que les faits que vous racontez sont trop monstrueux pour être crus" (Levi, 1989, p. 207).

Paris, le 25 mai 2002

RUSE-CEFRESS, Université de Picardie.

\section{Notes}

1 Ce travail aurait été impossible sans les informations qui m'ont été fournies par Andrzej Leder, philosophe, psychiatre et psychanalyste au Département de Philosophie de la Culture de L'Institut de Philosophie et Sociologie de l'Académie Polonaise des Sciences de Varsovie, les conseils 
bibliographiques et la lecture de son ouvrage, Un cri sans voix, de l'écrivain Henri Raczymow, l'aide bibliographique de Natalia Krynicka de la bibliothèque Medem, de Katy Hazan, auteur de Les orphelins de la Shoah, les maisons de l'espoir (1944-1960), de Cécilia Deshayes, étudiante de DEA au Département d'Ethnologie et Sociologie de l'Université de Picardie, d'Hervé Bagot, Architecte, Professeur à l'École d'Architecture de Nantes. Enfin, Roberta Shapiro, chargée de recherches à l'École d'Architecture de Nantes (Laboratoire, Architecture, Usage, Altérité) a bien voulu relire ce texte. Qu'ils soient tous ici vivement remerciés.

2 J'utilise ici les renseignements qui m'ont été fournis par Andrzej Leder (1967, p. 271), de Jan Jagielski et de Robert Pasieczny (1995).

3 Forteresse d'Hérode, les zélotes s'y réfugièrent après la prise de Jérusalem par les Romains et la destruction du Temple (70). Il est le symbole du suicide collectif de combattants qui choisirent la mort plutôt que l'esclavage.

4 Selon la rumeur qui courait alors à Varsovie, cette "opération de charme" du général Jaruselski était une surenchère vis-à-vis de Solidanorc soucieuse de rapprochement avec les Juifs.

5 Ce sont Lewartowski, Klepfisz, Wilner, Majerowicz, Plotnicka, Nyssenbaum, Zygielbojm, Korczak le pédagogue, Katzenelson, auteur de Le chant du peuple juif massacré, traduit par M. Novitch et S. Der (Ghetto Fighters House, 1983) et, dernièrement par Batia Baum: "Chant du peuple juif assassiné" publié dans la revue Caravanes, n. 7. Cf. Ringelblum (1995).

6 Suivant l'ouvrage Retour sur le ghetto de Varsovie, Marian Apfelbaum (2002) rappelle qu'il n'y a pas que les militants de gauche qui ont participé à la révolte du ghetto. Le ZZW ("Union militaire juive") a disparu "volontairement" de l'histoire parce qu'elle regroupait des hommes de droite mais aussi parce qu'elle avait été davantage soutenue par la résistance polonaise que le PPR (parti communiste). Je ne suis pas compétente pour juger de ces faits, je me contente ici de renvoyer à l'ouvrage et aux critiques éventuelles qui en sont ou en seront faites par les historiens.

7 Pour la distribution de pain, voir Hillel Seidman, Du fond de l'abîme, la photo - p. 486 - distribution de pain à toute personne qui se rendrait volontairement, à l'Umschlagplatz (place de transbordement), en vue 
de sa "réinstallation à l'est" (CDJC), Terre humaine, Plon, 1998. Noter également la couverture de la réédition de Chronique du ghetto de Varsovie (Ringelblum (1995). Ces chroniques enterrées dans deux endroits du ghetto ont été retrouvées en 1946 puis en 1950. Emmanuel Ringelblum a été assassiné sur les ruines du ghetto le 7 mars 1944.

8 Crée à l'initiative du "Comité citoyen pour la protection des cimetières et monuments de la culture juive", qui existe depuis 1981.

9 A une époque où, sous la présidence du général Jaruselski, comme tout projet de cette envergure, il avait dû passer par son secrétariat mais, selon la version officielle, il était né à l'initiative des "citoyens et autorités de Varsovie".

10 Je dois cette lecture à Hervé Bagot. Ceux qui ont vu le film de CostaGavras, Amen, se rappelleront "ces trains ouverts et fermés traversant impitoyablement lieux et images" (La lettre des amis de la CCE, n.38, avril, 2002) qui scandent une partie du film tiré de la pièce de R. Hochhuth, Le vicaire (Seuil, 1963).

11 C'est du moins, faute de commentaires comme nous en avons pour l'œuvre de Natan Rapoport par lui-même, ce que nous pouvons supposer.

12 Des découvertes similaires ont été faites par le photographe de Drancy.

13 Ces informations et celles qui vont suivre, ont été puisées par notre informateur polonais dans un ouvrage de Janusz Sujecki (1993, p. 2729). Au début des années 90, il y a eu un accord entre la ville et la Fondation Lauder pour un projet de reconstruction. Jusqu'à aujourd'hui, rien n'a été fait.

14 "Toutes les descriptions de la yidishe gas, la rue juive, insistent sur l'impression de foule. Ces quartiers sont à haute densité de population. De fait, un peu moins de 30\% des Juifs de Varsovie habitent dans des appartements d'une seule pièce, appartements surpeuplés, puisque, pour les trois quarts, ils abritent en moyenne trois ou quatre personnes... Les Polonais sont encore plus mal lotis" (Wieviorka, 1993-1994).

15 Les cahiers de médiologie, n. 7, "La confusion des monuments", "Le monument à l'épreuve du patrimoine".

16 Louise Merzeau, "Du monument au document", Cahiers de médiologie, n. 7. 
17 La réalisation en a été retardée parce que la Ville avait décidé de construire une maison sur la place Zygielbojm; ils ont donc dû attendre que la construction soit terminée pour entreprendre la compression. D'après Hanna Szmalenberg, l'inauguration date d'il y a quatre ou cinq ans.

18 Zygielbojm était un ancien membre du Judenrat (Voir Weinstock, 1999).

19 Voir à ce propos d'Arthur Danto (2000). Je reprendrai toute cette discussion dans un ouvrage en cours.

20 On sait que Gilles Deleuze nomme ce passé qui fait retour dans le présent, la deuxième synthèse du temps, la première étant celle de la répétition simple, et la troisième, celle que l'on vient de décrire à travers l'exemple de la Shoah (la catastrophe) justement (cf. Différence et répétition, 1969).

21 Il était installé dans un ensemble d'habitations non achevé destiné à la gendarmerie. La question de son classement aux Monuments Historiques, au double titre de première construction collective à caractère social due à Lodz et Beaudoin, et du camp installé dans ses murs, choque la philosophe qui lui oppose Verdun, éloigné de toute habitation et surtout conçu sur la base de traces encore présentes, de photos... Ne répondant plus du tout aux normes contemporaines, à Drancy, vivent toujours cinq cents personnes qui craignent d'être expulsées par le classement. Ce dernier ne concerne que les façades, les toitures, les cages d'escalier, les sous-sols mais pas les caves - celles-ci appartiennent aux habitants qui peuvent, s'ils le désirent, enlever les traces et inscriptions, qui seraient seules conservées, alors, par le photographe.

\section{Métropolis.}

23 Voir ce qu'elle écrivait déjà dans L'allégorie du patrimoine (1999).

24 Concernant spécifiquement la question du témoignage, Annette Wieviorka a montré, dans L'ère du témoin (2002), combien la massmédiatisation de l'Holocauste (cf. La liste de Schindler, film de Steven Spielberg, et à la suite, ses enquêtes auprès des «survivants»), amenait à remplacer le travail de l'historien par la «vérité» vécue du témoin. Il ne s'agit pas de récuser une sociologie du «vécu» dont la «vérité» n'est pas moins grande mais d'affirmer qu'elle est d'un autre ordre. Les mémoriaux déclinent une sensibilité qui ne peut être confondue avec la preuve. Cependant, il ne faut pas pour autant oublier qu'il y a aussi un rituel de la preuve ainsi que nous l'affirmons dans le corps du texte qui montre bien que, d'une manière ou d'une autre on échappe guère à ces formes culturelles propres à toute société et à toute forme de savoir. 
«L'américanisation» contemporaine de l'Holocauste est un phénomène collectif intéressant en ce qu'il montre combien les USA sont désormais engagés, même avec la meilleure volonté apparente, dans une pratique d'appropriation abusive et de colonisation culturelle. Sans aller jusquelà, peut-être faut-il imputer cette opération à la culpabilité de n'avoir rien su ou rien fait durant les événements mais aussi, comme on le voit de manière évidente actuellement à propos du 11 sept., par une adhésion toute religieuse à la logique du Bien et du Mal. A cela il faut ajouter la proximité culturelle - celle de gens cultivés appartenant pour la plupart à la classe moyenne -, alimentée par l'arrivée importante des intellectuels fuyant l'Allemagne nazie. Pour cette question, voir le passionnant ouvrage de Peter Novick (1999).

25 Il légitime son point de vue en faisant référence à Pierre Nora, ce qui me semble abusif car ce dernier prend bien soin de distinguer realia et templum, pour différencier histoire et mémoire, sans émettre aucun jugement de valeur à l'égard des faits eux-mêmes. On n'entrera pas ici dans le détail d'un débat qui fait rage aux USA comme en France. (Cf. Le Débat, n. 98, 1998, Peter Novick (1999) et son compte-rendu critique (2001) par Maurice Kriegel).

26 A James E. Young, révulsé à l'idée de toilettes près des chambres à gaz, on a envie de répondre prosaïquement, selon la tradition juive également, que "la vie continue"...

27 Le mémorial à S. Zygielbojm a peut-être été détaché du «chemin du souvenir», dont il devait faire partie au départ, parce qu'il exprime une culpabilité contemporaine, manifeste surtout aux Etats-Unis qui considère que les Alliés auraient dû accéder à sa demande. On peut dire, désormais que, si les bombardements des voies de chemin de fer ou d'Auschwitz auraient certes prouvé aux Juifs qu'ils n'étaient pas les abandonnés de la terre, il n'en reste pas moins, ainsi que le souligne P. Novick (1999), que cela n'aurait «objectivement» servi à rien.

28 Voir surtout la première partie: "Dégagement de la question de la vérité, l'expérience de l'art".

29 On a vu, à nouveau, ce même groupe des "Arts de la Rue" surplomber le flot des participants à la dernière manifestation du 1er mai 2002 durant tout le parcours de la République à la Nation.

30 Enquête sur le quartier Saint-Paul (Ostrowetsky, 1994). 
31 Wolfgang Sofsky (1995, p. 250) cité à propos du "musulman" définit comme figure anthropologique du pouvoir absolu par Giorgio Agamben (1999, p. 57).

\section{Bibliographie}

AGAMBEN, Giorgio. Ce qui reste d'Auschwitz. [1998]. Paris: Rivages, 1999.

APFELBAUM, Marian. Retour sur le ghetto de Varsovie. Paris: Odile Jacob, 2002.

BATESON, Gregory. Vers une écologie de l'esprit. Paris: Seuil, 1977. Tome 1.

BENSOUSSAN, Georges. Génocide pour mémoire. Paris: Éditions du Félin, 1989.

CHOAY, Françoise. L’allégorie du patrimoine. Paris: Seuil, 1992, 1996, 1999.

DANTO, Arthur. L'art contemporain et la culture de l'histoire. Paris: Seuil, 1997, 2000.

DELEUZE, Gilles. Différence et répétition. Paris: PUF, 1969.

GADAMER, Hans Georg. Vérité et méthode. [1960]. Paris: Seuil, 1976, 1996.

GARFINKEL, Harold. Studies in Ethnometodology. New York: Englewood Cliffs, 1967.

HALBWACHS, Maurice. La mémoire collective. Paris: PUF, 1950; Albin Michel, 1997.

HATZFELD, Jean. Dans le nu de la vie. Paris: Seuil, 2000.

HAZAN, Katy. Les orphelins de la Shoah, les maisons de l'espoir (19441960). Paris: Les Belles Lettres, 2001.

HEINICH, Nathalie. Ce que l'art fat à la sociologie. Paris: Minuit, 1998.

HOCHHUTH, R. Le vicaire. Paris: Seuil, 1963.

JAGIELSKI, Jan; PASIECZNY, Robert. A Guide to Jewish Warsaw. Warsaw, 1995. (Library of “Our Roots”, v. 1).

KATZENELSON, Jizchaq. Le chant du peuple juif massacré. Traduit par M. Novitch et S. Der. Israel: Ghetto Fighters House, 1983.

. Chant du peuple juif assassiné. Traduit par Batia Baum. Caravanes, Paris, n. 7, 2001. 
KRIEGEL, Maurice. Trois mémoires de la Shoah: Etats-Unis, Israël, France [à propos de Peter Novick, L'holocauste dans la vie américaine]. Le débat, n. 117, nov./dec. 2001.

LE DÉBAT, n. 98, janv./fev. 1998. L'unicité de la Shoah: P. Bouretz, J. M. Chaumont, Richard Marienstras, Michel Wieviorka, Peter Novick.

LEDER, Andrzej. Guide Bleu de la Pologne. Paris: Hachette, 1967.

LEVI, Primo. Les naufragés et les rescapés. Paris: Gallimard, 1989.

LYOTARD, Jean-François. La condition post-moderne. Paris: Minuit, 1979.

MELOT, Michel. Le monument à l'épreuve du patrimoine, Les cahiers de médiologie, $\mathrm{n}^{\circ} 7$, La confusion des monuments, $1^{\text {er }}$ semestre, 1999. http:www.medialogie.org/collection/07_monuments/melot.pdf.

MERZEAU, Louise. Du monument au document. Cahiers de médiologie, $\mathrm{n}$. 7, La confusion des monuments, $1^{\text {er }}$ semestre, 1999. http:www.medialogie.org/collection/07_monuments/merzeau.pdf.

NOVICK, Peter. L'holocauste dans la vie américaine. Paris: NRF Gallimard, 1999.

OSTROWETSKY, Sylvia. La représentation et ses doubles. Communication, Information, Université Laval, v. 6, n. 2/3, 1984. . Quelqu'un ou le livre de Moïshe. Paris: Kimé, 1995.

. Suite sur la puissance des dispositifs spatiaux. In: FIGURES architecturales, formes urbaines. (Pellegrino ed.). Paris: Anthropos, 1994.

RACZYMOW, Henri. Un cri sans voix. Paris: Gallimard, 1985.

RIEGL, Aloïs. Le culte moderne des monuments. Paris: Seuil, 1984.

RINGELBLUM, Emmanuel. Chronique du ghetto de Varsovie. Paris: Payot, [1958] 1995.

RYMKIEWICZ, Jaroslaw Marek. La dernière gare. Préface de Henri Raczymow. Paris: Laffont, 1998.

SEIDMAN, Hillel. Du fond de l'abîme. Paris: Plon, 1998. (Collection Terre Humaine).

SOFSKY, Wolfgang. L'organisation de la terreur. Paris: Calmann-Lévy, 1995.

SUJECKI, Janusz. Prozna, la rue sauvée de Varsovie juive. Varsovie: Ed. Ortis, 1993. 
WEINSTOCK, N. (Org.). Chroniques du désastre. Textes présentés et traduits du yiddish par N. Weinstock, préface de G. Bensoussan. Genève: Métropolis, 1999.

WIEVIORKA, Annette. L'ère du témoin. Paris: Hachette, 2002.

. Les Juifs de Varsovie à la veille de la Seconde Guerre mondiale. Les cahiers de la Shoah, éditions Liana Lévi, 1993-1994.

YOUNG, James E. Inscriptions juives dans l'espace. Pardès, Paris, n. 13, 1991. 